\begin{tabular}{|c|c|}
\hline Title & 3-D topology optimization of magnetic cores for wireless power transfer with double sided winding coils \\
\hline Author(s) & Otomo, Y oshitsugu; Igarashi, Hajime \\
\hline Citation & $\begin{array}{l}\text { International journal of applied electromagnetics and mechanics, 60(S1), S115-S123 } \\
\text { https://doi.org/10.3233/JA E-191110 }\end{array}$ \\
\hline Issue Date & $2020-03-02$ \\
\hline DOC URL & http:/hdl.handle.net/2115/76839 \\
\hline Rights & The final publication is av ail able at IOS Press through http://dx.doi.org/10.3233/JA E-191110 \\
\hline Type & article (author version) \\
\hline File Information & Full_paper_final_submission_IJAEM.pdf \\
\hline
\end{tabular}

Instructions for use 


\title{
3-D Topology Optimization of Magnetic Cores for Wireless Power Transfer with Double-Sided Winding Coils Yoshitsugu OTOMO* and Hajime IGARASHI*
}

* Graduate School of Information Science and Technology, Hokkaido University, Sapporo, 060-0814, JAPAN

\begin{abstract}
This paper presents three-dimensional topology optimization of magnetic cores for wireless power transfer with double-sided winding coils. In this optimization, the magnetic core shape is represented by the linear combination of the normalized Gaussian functions. The core shape is optimized so that the coupling coefficient between the transmitting and receiving coils is maximized. Moreover, robustness against misalignment between the coils is taken into account in the optimization. It is found that the optimized core shape effectively increases the net magnetic flux interlinked with the receiving coil, and outperforms the conventional core shapes.
\end{abstract}

Keywords: Electric vehicle, Genetic algorithm, Robust optimization, Topology optimization, Wireless power transfer.

\section{INTRODUCTION}

In recent years, electric vehicles (EVs) have been rapidly spreading because of environmental concerns and their low fuel consumption. Currently, one has to connect an electric cable to the EV body for battery charging. Because such operation would include accidental risks and needs some physical labour, it is expected to be replaced with wireless power transfer (WPT) [1]-[9]. The WPT systems have following advantages: 1) power can be supplied without physical connection, 2) it is safe even in dirty and wet environments, 3) maintenance is easy. The WPT device for EVs is required to have high efficiency, and simultaneously the magnetic cores placed in the vicinity of the transmitting and receiving coils to improve the efficiency should be as small as possible for reduction of cost and size. For such WPT devices, bar shaped and H-shaped cores with double-sided winding coils have been proposed $[5,6]$. To further improve the efficiency, design optimization of the WPT device based on field computation is effective.

In contrast to the conventional parameter optimization, the topology optimization allows us to obtain optimized shapes without introducing design parameters [10]-[13]. In particular, the on / off method based on the normalized Gaussian network (NGnet) has been shown effective for the design of rotating machines and antennas [10, 11]. This optimization method has been applied to two-dimensional optimization problems. In this paper, we extend the above method to three-dimensional optimization for design of the magnetic core of a WPT device with doublesided winding coils. In the present optimization, the core shape is optimized so that the coupling coefficient of the WPT device is maximized. Moreover, we have to design the robust WPT device against the misalignment between the transmitting and receiving coils because it is difficult to perfectly align the position of EVs to the transmitting coil [7]-[9]. Thus, we propose the robust optimization method for the WPT device considering misalignment between the transmitting and receiving coils.

\section{OPTIMIZATION METHOD}

In the topology optimization of a WPT device using NGnet which is schematically shown in Fig. 1, the material attribute in the design region is determined from the value of the shape function defined by 


$$
y(\boldsymbol{x})=\sum_{i=1}^{N} w_{i} b_{i}(\boldsymbol{x})
$$

where $w_{i}, \boldsymbol{x}$ and $N$ denote the weighting coefficient, center of finite element and number of Gaussian functions, respectively, and $b_{i}(\boldsymbol{x})$ denotes the normalized Gaussian function given by

$$
\begin{gathered}
b_{i}(\boldsymbol{x})=G_{i}(\boldsymbol{x}) / \sum_{j=1}^{N} G_{j}(\boldsymbol{x}) \\
G_{i}(\boldsymbol{x})=\frac{1}{(2 \pi)^{\frac{3}{2}} \sigma^{3}} \exp \left\{-\frac{1}{2 \sigma^{2}}\left|\boldsymbol{x}-\boldsymbol{x}_{i}\right|^{2}\right\}
\end{gathered}
$$

where $\sigma$ denotes the standard deviation whose value is set to $\sigma=20.0 \mathrm{~mm}$ in the following optimization examples and $\boldsymbol{x}_{i}$ is the center of Gaussian basis $G_{i}(\boldsymbol{x})$. The material attribute $M_{e}$ of finite element $e$ in the design region is determined from the shape function $y(\boldsymbol{x})$ as follows:

$$
M_{e}=\left\{\begin{array}{cc}
\text { ferrite } & y(\boldsymbol{x}) \geq 0 \\
\text { air } & y(\boldsymbol{x})<0
\end{array}\right.
$$

Finally, we solve the following magnetostatic equation to evaluate the objective function.

$$
\operatorname{rot} v(\operatorname{rot} \boldsymbol{A})=\boldsymbol{J}
$$

where $v, \boldsymbol{A}$ and $\boldsymbol{J}$ denote the magnetic reluctivity, vector potential and current density, respectively. The FE discretization of (5) leads to

$$
\sum_{j} A_{j} \int_{\Omega} \operatorname{rot} \boldsymbol{N}_{i} v\left(\operatorname{rot} \boldsymbol{N}_{j}\right) d \Omega=\int_{\Omega_{\text {coil }}} \boldsymbol{N}_{i} \cdot \boldsymbol{J} d \Omega
$$

where $\boldsymbol{N}_{i}$ denotes the vector interpolation function. In the on/off method based on NGnet, the magnetic reluctivity in (6) is determined from (4).

In the optimization, $\boldsymbol{w}=\left\{w_{i} \mid i=1,2, \ldots, N\right\}$ is determined so as to maximize the objective function by micro genetic algorithm $(\mu \mathrm{GA})[14]$ subjected to given constraints. Namely, the topology optimization is reduced to determination of the array of real numbers $\boldsymbol{w}$.

\section{OPTIMIZATION PROBLEM}

We want to optimize the magnetic core shape to maximize the efficiency of a WPT device keeping the volume of the magnetic core as small as possible for reduction of cost and size. Moreover, the efficiency should be kept as high as possible even though misalignment between the transmitting and receiving coils exists. Thus, to obtain the optimal core shape which has good tolerance to misalignment, we maximize the magnetic coupling for several vertical misalignment patterns. The optimization problem considering the robustness is defined by 


$$
\max _{\boldsymbol{w}} F(\boldsymbol{w}), \quad F(\boldsymbol{w})=\frac{1}{N_{\mathrm{p}}} \sum_{i=1}^{N_{\mathrm{p}}} k_{i}(\boldsymbol{w}), \text { sub. to } \Omega_{\mathrm{M}} \leq \frac{\Omega_{\mathrm{D}}}{2}
$$

where $k_{i}(\boldsymbol{w})\left(i=1,2, \ldots, N_{\mathrm{p}}\right), \Omega_{\mathrm{M}}$ and $\Omega_{\mathrm{D}}$ denote the coupling coefficient for the $i$-th misalignment pattern, total volume of the magnetic core and volume of the design region, respectively. In the problem defined by (7), the average of the coupling coefficient is considered for simplicity, while we can modify the problem by introducing different weighting coefficients for $k_{i}(\boldsymbol{w})$. Assuming that the transmitting and receiving coils have same selfinductance, the coupling coefficient $k_{i}(\boldsymbol{w})$ is obtained from the finite element analysis as follows:

$$
k_{i}(\boldsymbol{w})=\frac{\int_{\Omega_{\mathrm{coil} 2}} \boldsymbol{A}_{i}(\boldsymbol{w}) \cdot \boldsymbol{J}_{2} d \Omega}{\int_{\Omega_{\mathrm{coil} 1}} \boldsymbol{A}_{i}(\boldsymbol{w}) \cdot \boldsymbol{J}_{1} d \Omega}
$$

where $\boldsymbol{A}_{i}(\boldsymbol{w}), \boldsymbol{J}_{1}$ and $\boldsymbol{J}_{2}$ denote the vector potential for the $i$-th misalignment pattern and unit vectors parallel to the currents along the transmitting and receiving coils, respectively. Note that the vector potential $\boldsymbol{A}_{i}(\boldsymbol{w})$ is implicit function of the weighting coefficient. To solve the optimization problem using $\mu \mathrm{GA}$ [14], the number of individuals is set to 5 and the evolution process is continued for 400 generations. In this setting, it takes about 2.5 days to obtain the optimization results using the Intel Xeon $C P U(3.5 \mathrm{GHz}, 5$ cores).

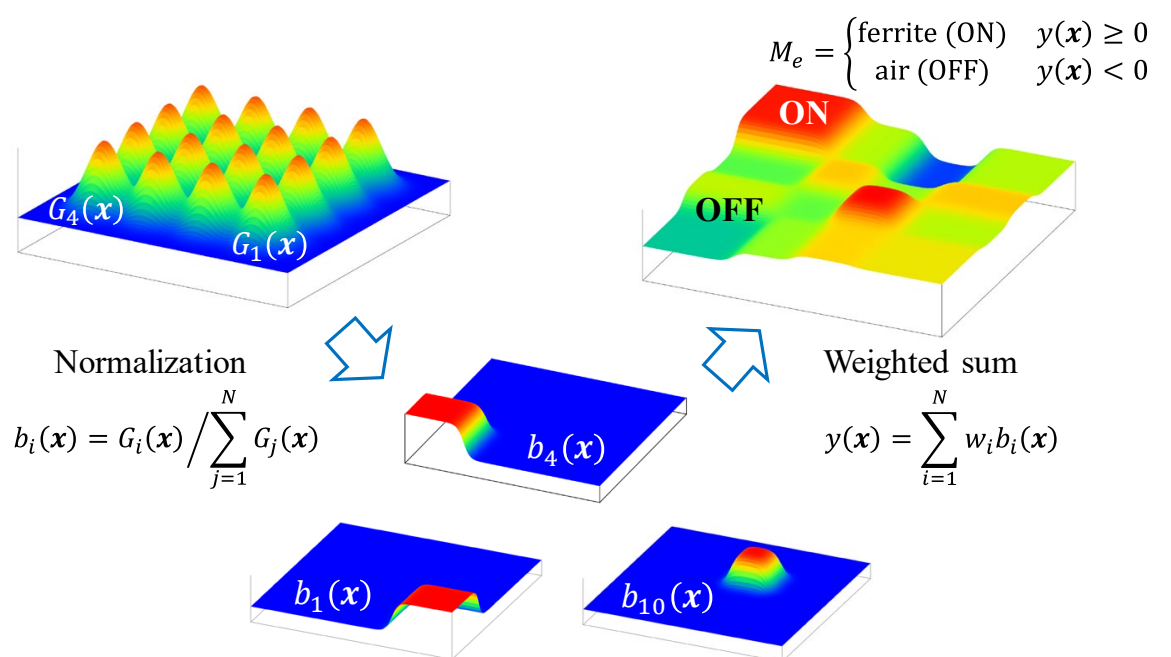

Figure 1: On / off method using normalized Gaussians (2D example)

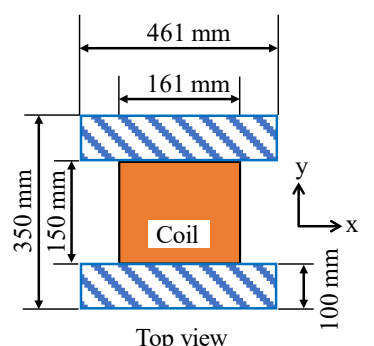

Top view

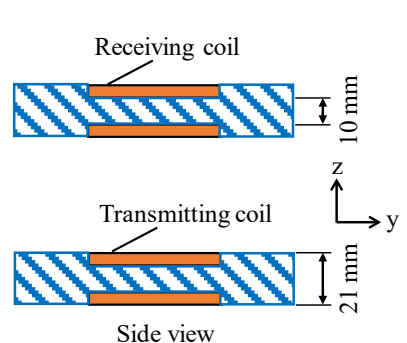

Side view

: Design region

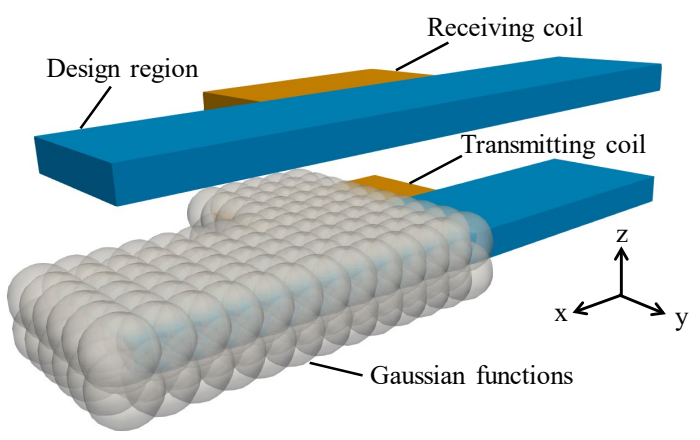

Figure 2: Optimization model

Figure 3: Distribution of Gaussian functions 
Table 1: Specifications of double-sided winding coils

Strand radius

Number of strands

Number of turns

Relative permeability of magnetic core

Driving frequency

Lateral misalignment ( $x$-axis)

Forward misalignment ( $y$-axis)

Air gap length
$0.15 \mathrm{~mm}$

100

3 parallels $\times 15$ turns

1500

$85 \mathrm{kHz}$

$300 \mathrm{~mm}(\mathrm{MAX})$

$60 \mathrm{~mm}(\mathrm{MAX})$

$70+30(-15) \mathrm{mm}$

\section{OPTIMIZATION RESULTS}

The optimization model of a WPT device with double-sided windings for EVs is shown in Fig. 2 and table 1 summarizes the coil parameters. To shape the transmitting and receiving coils with mirror symmetries in $x$ and $z$ directions, 196 Gaussian functions are uniformly deployed so that the 1/4 fraction of the domain of transmitting coil is covered by the support of the Gaussians as shown in Fig. 3, where the spheres whose radius is the standard deviation $\sigma$ are located at $\boldsymbol{x}_{i}(i=1,2, \ldots, N)$. Although the misalignment in the forward direction $(y$-axis $)$ of EVs is easily limited by using a wheel stopper, it is difficult to limit the misalignment in the lateral direction ( $x$-axis). For this reason, we consider the lateral misalignments in (7). Because the good tolerance is required with respect to the lateral misalignment, we consider three different misalignments $0 \mathrm{~mm}, 150 \mathrm{~mm}, 300 \mathrm{~mm}$, which correspond to $i=1,2,3$ in (7), respectively. To verify effectiveness of the robust optimization, we have also performed optimization without considering misalignment such that only $k_{1}(w)$ is considered in (7).

The optimized results with and without considering misalignment, represented by "Optimized (Robust)" and “Optimized (Non-Robust)", respectively, are shown in Fig. 4 (a), (b). For comparison, the bar shaped and Hshaped cores, which are discussed in [5] and [6], are shown in Fig. 4 (c), (d). All the cores in Fig. 4 have the same ferrite volume. One of the remarkable features in the optimized results is the existence of the holes in the core. The core length in $x$-direction in (a) is longer than that in (b) because of the robustness against the misalignment. The coupling coefficients are plotted against the degree of misalignment in Fig. 5. We can see that the optimized WPT with considering misalignment in Fig. 4 (a) has the good tolerance not only in lateral misalignment but also in forward misalignment and air gap. The coupling coefficient of the optimized WPT in Fig. 4 (a) with misalignment of $300 \mathrm{~mm}$ is larger by about $1.5 \%$ and $3 \%$ than that of the bar shaped and H-shaped cores. In contrast, the optimized WPT in Fig. 4 (b) without considering misalignment has the smaller coupling coefficient in comparison that in (a) when the misalignment is greater than $100 \mathrm{~mm}$. In particular, the its coupling coefficient with misalignment of $300 \mathrm{~mm}$ is smaller by about $2 \%$ than that of the bar shaped core. To interpret the difference in the performance of the WPTs in Fig. 4 (a) and (b), the flux distributions with lateral misalignment are compared in Fig. 6. It can be seen that there are flux linkages between the edge of the upper core and the center of the lower core in Fig. 6 (a) whereas no flux linkage can be found there in Fig. 6 (b). 
In order to study the dependence of the solution on the initial individuals in $\mu \mathrm{GA}$, we perform the optimization starting from five different sets of individuals generated by changing random seed. The convergence histories of $\mu \mathrm{GA}$ with and without considering misalignment are plotted for the five cases in Fig. 7. In addition, the standard deviations $\sigma_{a}$ and $\sigma_{b}$ of the objective function at the 400-th generation are also shown in Fig. 7. We can see that the optimization results have almost the same performance because the standard deviations among them are less than $1 \%$.

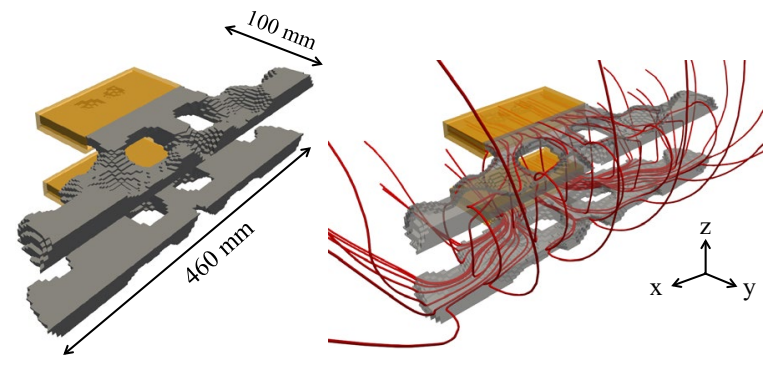

(a) Optimized core considering misalignment

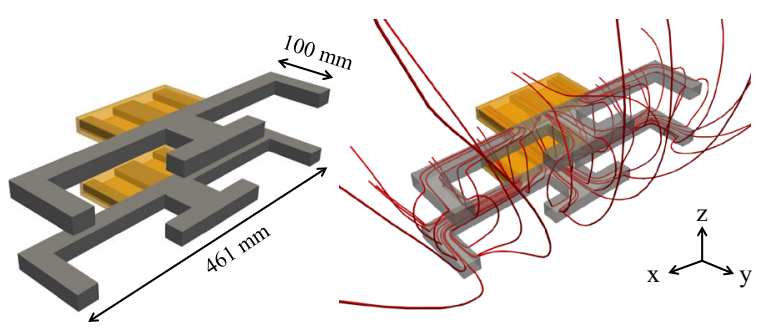

(c) Bar shaped [5]

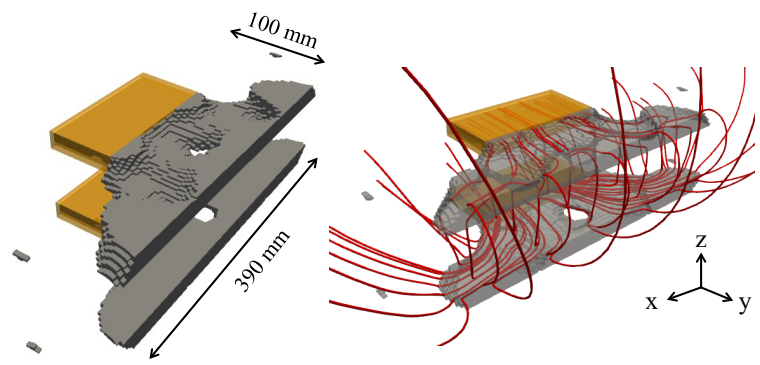

(b) Optimized core without considering misalignment

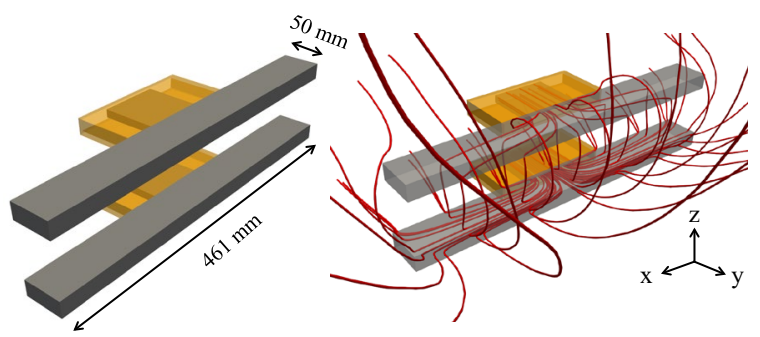

(d) H-shaped [6]

Figure 4: WPT cores with same ferrite volume (1/2 fraction of WPT is shown).

The magnetic flux lines are drawn in the right figures.

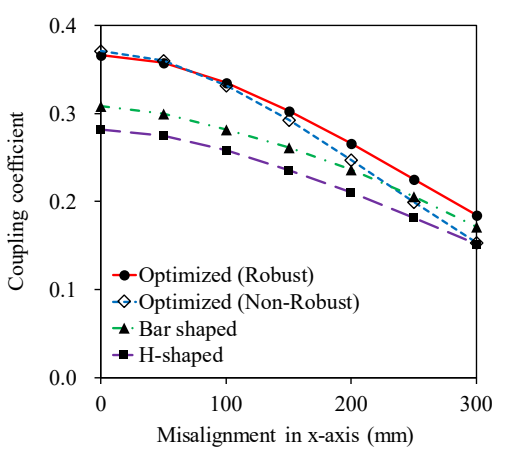

(a) Lateral misalignment with air gap $=70 \mathrm{~mm}$

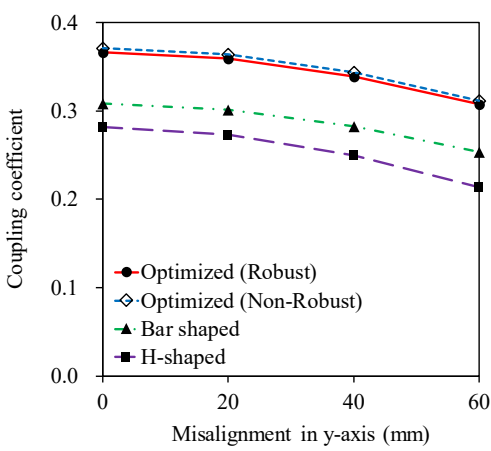

(b) Forward misalignment with air gap $=70 \mathrm{~mm}$

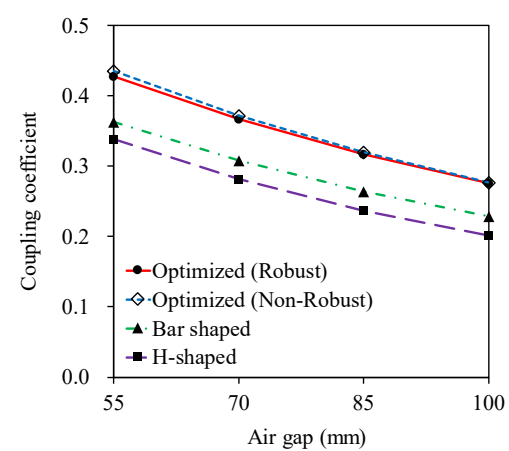

(c) Air gap length with alignment 




(a) Optimized core considering misalignment

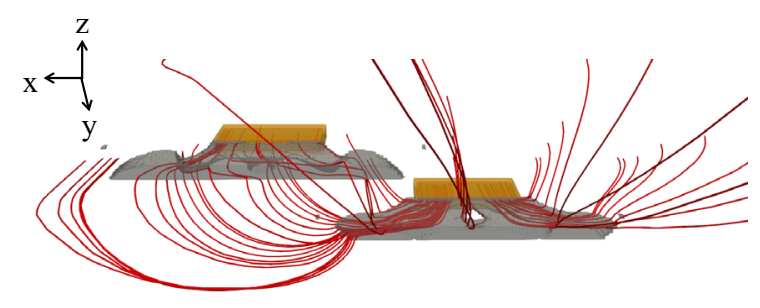

(b) Optimized core without considering misalignment

Figure 6: Comparison results of flux distributions with lateral misalignment $=300 \mathrm{~mm}$

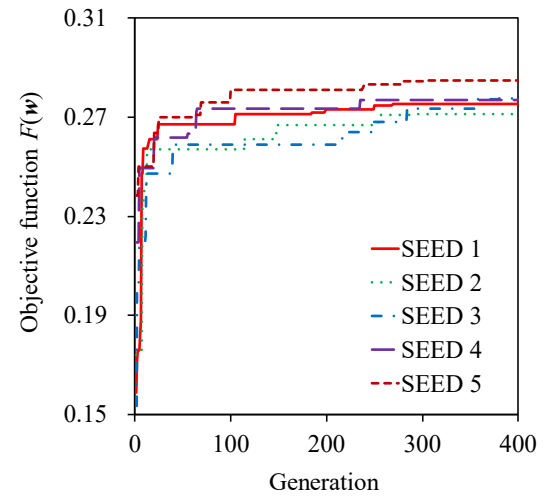

(a) Considering misalignment $\left(\sigma_{a}=4.34 \times 10^{-3}\right)$

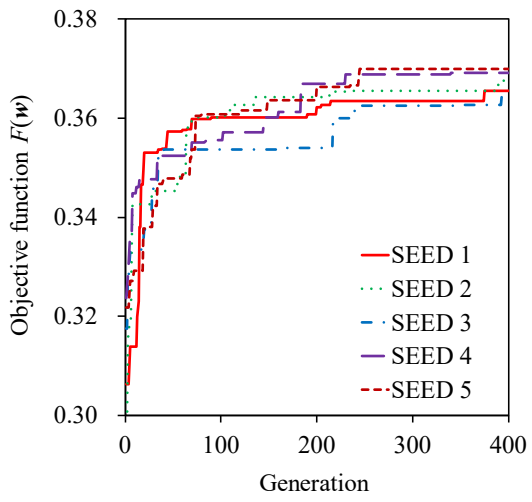

(b) Without considering misalignment $\left(\sigma_{b}=2.11 \times 10^{-3}\right)$

Figure 7: Convergence histories of $\mu G A$

\section{CONSIDERATION OF LEAKAGE FLUX}

The compliance with the guidelines to limiting exposure of magnetic and electric fields has to be contemplated in the design of WPT device. The International Commission on Non-Ionizing Radiation Protection (ICNIRP) requires that the general public should not be exposed to average RMS flux densities higher than $27 \mu \mathrm{T}$ in the frequency range of $3 \mathrm{kHz}-10 \mathrm{MHz}$ [15]. To verify if the WTPs meet the guidelines, we have compared the leakage flux densities of the optimized and conventional cores at $1 \mathrm{~kW}$ power transfer. The output power $P_{\text {out }}$ is evaluated from [16]

$$
P_{\text {out }}=\omega I_{1}^{2} k^{2} L_{1} Q
$$

where the $\omega, I_{1}, k, L_{1}$ and $Q$ denote the angular frequency, RMS value of the current in the transmitting coil, coupling coefficient, self-inductance of the transmitting coil and quality factor of the receiving coil, respectively. The computed leakage flux densities are plotted against the distance from the center $O$ of the WPT coil in Fig. 8. We can see that the leakage flux density of the optimized core shown in Fig. 4 (a) has almost the same value as that of the conventional core shapes in the all conditions. It is concluded from these results that the optimized core has almost the same exposure level as the conventional cores. 


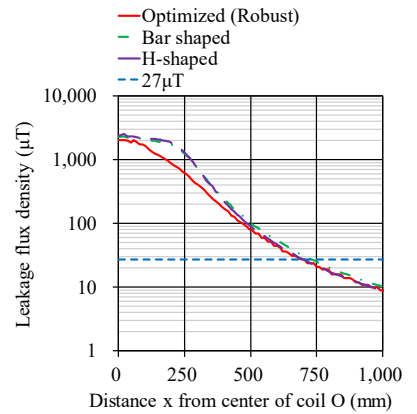

(a) Lateral direction with alignment

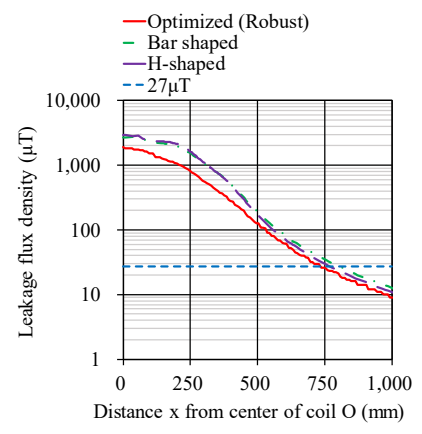

(c) Lateral direction with misalignment $=150 \mathrm{~mm}$

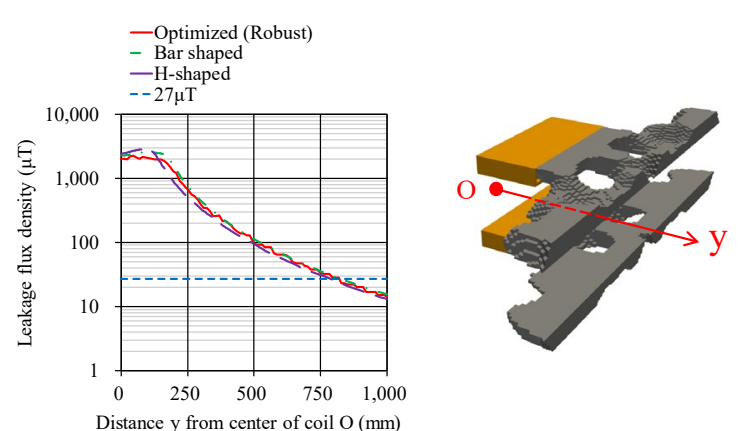

(b) Forward direction with alignment

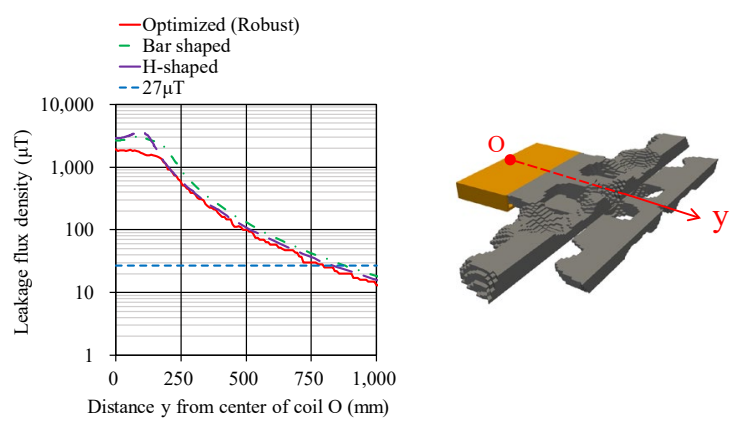

(d) Forward direction with misalignment $=150 \mathrm{~mm}$

Figure 8: Simulated leakage flux density

\section{CONCLUSION}

In this paper, we have proposed a three-dimensional topology optimization method to effectively improve the efficiency of the WPT device. It is shown that the optimized core shape obtained by the proposed method improves the coupling coefficient. In particular, it is found that the WPT device with the magnetic core optimized considering misalignment outperforms the conventional WPTs with respect to the coupling efficiency. Moreover, the optimized WPT has almost the same exposure level as the conventional WPTs. In future, we plan to manufacture the optimized core to verify its performance by experiments.

\section{ACKNOWLEDGEMENT}

This study was supported in part by JSPS KAKENHI Grant Numbers JP18K18840 and JP18H01664.

\section{REFERENCES}

[1] Y. Nagatsuka, N. Ehara, Y. Kaneko, S. Abe and T. Yasuda, Compact contactless power transfer system for electric vehicles, International Power Electronics Conference (IPEC), pp. 807-813, 2010.

[2] A. A. S. Mohamed, S. An and O. Mohammed, Coil design optimization of power pad in IPT system for electric vehicle applications, IEEE Trans. Magn., vol. 54, no. 4, Art. no. 9300405, 2018.

[3] T. Yilmaz, N. Hasan, R. Zane and Z. Pantic, Mult-objective optimization of circular magnetic couplars for wireless power transfer applications, IEEE Trans. Magn., vol. 53, no. 8, Art. no. 8700312, 2017.

[4] M. Budhia, J. T. Boys, G. A. Covic and C. Y. Huang, Development of a single-sided flux magnetic coupler for electric vehicle IPT charging systems, IEEE Trans. Ind. Electron., vol. 60, no. 1, pp. 318-328, 2013.

[5] M. Budhia, G. Covic and J. Boys, A new IPT magnetic coupler for electric vehicle charging systems, 36th Annual Conference on IEEE Industrial Electronics Society (IECON 2010), pp. 2487-2492, 2010. 
[6] M. Chigira, Y. Nagatsuka, Y. Kaneko, S. Abe, T. Yasuda and A. Suzuki, Small-size light-weight transformer with new core structure for contactless electric vehicle power transfer system, Energy Conversion Congress and Exposition (ECCE), pp. 260-266, 2011.

[7] G. Yang, S. Dong, C. Zhu, R. Lu, G. Wei and K. Song, Design of a high lateral misalignment tolerance magnetic coupler for wireless power transfer systems, IEEE PELS Workshop of Emerging Technologies : Wireless Power Transfer (WoW), pp. 34-39, 2017.

[8] S. Bandyopadhyay, V. Prasanth, L. R. Elizondo and P. Bauer, Design considerations for a misalignment tolerant wireless inductive power system for electric vehicle (EV) charging, 19th European Conference on Power Electronics and Applications (EPE'17 ECCE Europe), pp. 1-10, 2017.

[9] M. Mohammad, S. Choi, M. Z. Islam, S. Kwak and J. Baek, Core design and optimization for better misalignment tolerance and higher range of wireless charging of PHEV, IEEE Trans. Transport. Electrific., vol. 3, no. 2, pp. 445-453, 2017.

[10] T. Sato, K. Watanabe and H. Igarashi, Multimaterial topology optimization of electric machines based on nomalized gaussian network, IEEE Trans. Magn., vol. 51, no. 3, Art. no. 7202604, 2015.

[11] T. Mori, R. Murakami, Y. Sato, F. Campelo and H. Igarashi, Shape optimization of wideband antennas for microwave energy harvesters using FDTD, IEEE Trans. Magn., vol. 51, no. 3, Art. no. 8000804, 2015.

[12] Y. Okamoto, Y. Tominaga, S. Wakao and S. Sato, Topology optimization of rotor core combined with identification of current phase angle in IPM motor using multistep genetic algorithm, IEEE Trans. Magn., vol. 50, no. 2, Art. no. 7017904, 2014.

[13] K. Watanabe, T. Suga and S. Kitabatake, Topology optimization based on the on/off method for synchronous motor, IEEE Trans. Magn., vol. 54, no. 3, Art. no. 7201104, 2018.

[14] C. A. C. Coello and G. T. Pulido, A micro-genetic algorithm for multiobjective optimization, International Conference on Evolutionary Multi-Criterion Optimization (EMO 2001), pp. 126-140, 2001.

[15] Guidelines for limiting exposure to time-varying electric and magnetic fields (1 Hz-100 kHz), Health Physics, vol. 99, no. 6, pp. 818836, 2010.

[16] O. H. Stielau and G. A. Covic, Design of loosely coupled inductive power transfer systems, International Conference on Power System Technology (PowerCon 2000), pp. 85-90, 2000. 